Research Article

\title{
Outage Constraint Robust Cooperative Beamforming and Jamming Design for Secure AF Relay Networks
}

\author{
Jinxin Zhu $\mathbb{D}$ and Jun Shao \\ School of Information Technology, Yancheng Institute of Technology, Yancheng 224051, China \\ Correspondence should be addressed to Jinxin Zhu; zjx@ycit.edu.cn
}

Received 18 August 2021; Revised 3 October 2021; Accepted 20 October 2021; Published 5 November 2021

Academic Editor: Jun Li

Copyright ( 92021 Jinxin Zhu and Jun Shao. This is an open access article distributed under the Creative Commons Attribution License, which permits unrestricted use, distribution, and reproduction in any medium, provided the original work is properly cited.

\begin{abstract}
This paper investigates a joint robust scheme in a secrecy relay network where distributed relays perform cooperative beamforming and a friendly jammer transmits jamming signal to enhance the information security. Specifically, we consider the outage constraint secrecy rate maximization design with imperfect channel state information. Through semidefinite relaxation and onedimensional search, we propose a two-layer optimization method to solve the nonconvex problem. In addition, the Bernstein-type inequality and large deviation inequality are utilized to convert the probabilistic constraint. Simulation results demonstrate the performance of the proposed design.
\end{abstract}

\section{Introduction}

Physical layer security is considered as a promising technique in wireless networks to prevent illegitimate eavesdropping on the confidential message. Recently, cooperative transmission using relays to improve the physical layer security in wireless network has attracted increasing attention [1], while the cooperative beamforming (CB) and cooperative jamming (CJ) are the most useful ways for improving the security in relay networks [2].

Specifically, in [3], the authors proposed a joint CB and jamming design to provide Bob with certain quality of service (QoS). In [4], the authors proposed a joint $\mathrm{CB}$, jamming and power allocation to secure amplify-and-forward (AF) relay system, and was extend in [5] with considering hybrid opportunistic relaying and jamming, while in [6], the authors proposed a jamming scheme in secure AF relay system considering two-slot eavesdropping. Recently in [7], the authors proposed a destination-aided CJ scheme for the AF relay system when the relay is untrusted.

The above works assumed that the perfect channel state information (CSI) can be obtained. In practice, it may be difficult to obtain the perfect CSI due to channel estimation and quantization errors. To handle this obstacle, two kinds of robust secrecy design, e.g., the worst case (WC) design and the outage constraint (OC) design have been widely investigated. The WC secrecy design, which assumes that the CSI errors lie in a deterministic region, is an absolute safe design and the performance may be conservative. On the other hand, the OC method is a better choice when the CSI errors have certain probabilistic properties [8]. Specifically, the OC secrecy design was investigated for multiple-input single-output (MISO) system in [9-12] and for multipleinput multiple-output (MIMO) system in [13-15], respectively. Among these works, the Bernstein-type inequality (BTI) and large deviation inequality (LDI) are widely used to convert the probabilistic constraint into convex constraint.

However, all of these works investigated the direct communication scenario, and the OC secrecy design in relay network with friendly jammer has not been investigated yet. Motivated by this observation, in this paper, we concentrate on the OC robust design in friendly jammer-assisted secrecy AF relay networks. Specifically, assuming that the CSI error can be modeled as certain probabilistic distribution, we investigate the outage constraint secrecy rate maximization (OCSRM) problem, via jointly optimizing the $\mathrm{CB}$ and jamming covariance. Here, we model the CSI uncertainty level as a function of the signal-to-noise ratio (SNR), which 
rarely appears in related works. To solve the formulated nonconvex problem, we derive a safe approximation of the outage secrecy rate, and then a two-layer optimization algorithm, which consists of one-dimensional search and semidefinite relaxation (SDR), was proposed. Furthermore, the probabilistic constraints are transformed by the BTI and LDI, respectively. Simulation results demonstrate the performance of the proposed method.

1.1. Notations. Throughout this paper, boldface lowercase and uppercase letters denote vectors and matrices, respectively. The transpose, conjugate transpose, and trace of matrix $\mathbf{A}$ are denoted as $\mathbf{A}^{T}, \mathbf{A}^{H}$, and $\operatorname{Tr}(\mathbf{A})$, respectively. $\mathbf{a}=\operatorname{vec}(\mathbf{A})$ denotes to stack the columns of matrix $\mathbf{A}$ into a vector $\mathbf{a} . \mathbf{A} \geqslant \mathbf{0}$ indicates that $\mathbf{A}$ is a positive semidefinite matrix. denotes the Frobenius norm. $\odot$ denotes elementwise product. $\mathbf{D}(\mathbf{a})$ represents a diagonal matrix with $\mathbf{a}$ on the main diagonal. $\mathbf{I}$ is an identity matrix with proper dimension. $\operatorname{Re}\{a\}$ denotes the real part of a complex variable $a$. $\mathscr{C} \mathcal{N}(\mathbf{0}, \mathbf{I})$ denotes a circularly symmetric complex Gaussian random vector with mean $\mathbf{0}$ and covariance $\mathbf{I}$. $\lambda_{\max }(\mathbf{A}, \mathbf{B})$ denotes the maximum generalized eigenvalue of matrix pair $(\mathbf{A}, \mathbf{B})$. $\mathbb{E}$ denotes the mathematical expectation.

\section{System Model and Problem Formulation}

2.1. System model. We consider a cooperative relay network as shown in Figure 1, in which Alice communicates with Bob with the help of $M$ relays and a friendly jammer. The set of Eves is denoted as $\mathscr{K} \triangleq\{1, \ldots, K\}$. We assume that no direct link exists between Alice and Bob or between Alice and Eves. Each node is equipped with a single antenna except the jammer, which is equipped with $N_{j}$ antennas. All the channels are flatfading Rayleigh distribution. Specifically, the channel between Alice to relays, between relays to Bob and Eves, between the jammer to Bob and Eves are denoted by $\mathbf{h} \in \mathbb{C}^{M}, \mathbf{g}_{b} \in \mathbb{C}^{M}$, $\mathbf{g}_{e, k} \in \mathbb{C}^{M}, \mathbf{g}_{b, j} \in \mathbb{C}^{N_{j}}$, and $\mathbf{g}_{k, j} \in \mathbb{C}^{N_{j}}$, respectively, and we assume that these channels undergo flat Rayleigh fading. Since the relays operate in a half-duplex mode with the AF scheme, one transmission round is composed of two phases.

In the first phase, Alice broadcasts its information $s$ which satisfies $\mathbb{E}\left[|s|^{2}\right]=1$ to the relays. Thus, the received signal at the relays is expressed as $\mathbf{y}_{r}=\sqrt{P_{s}} \mathbf{h} s+\mathbf{n}_{r}$, where $P_{s}$ is the transmit power at Alice and $\mathbf{n}_{r}$ is the additive noise at the relays with distribution $\mathscr{C} \mathcal{N}\left(\mathbf{0}, \sigma_{r}^{2} \mathbf{I}\right)$.

In the second phase, the relays employ the $\mathrm{CB}$ vector $\mathbf{w} \triangleq\left[w_{1}, \ldots, w_{M}\right]^{T} \in \mathbb{C}^{M}$ to forward the information to the Bob, which grants Eves the opportunity to overhearing. Thus, the signal transmitted by the relays is given by $\mathbf{x}=\mathbf{D}\left(\mathbf{y}_{r}\right) \mathbf{w}$.

To confuse Eves, the jammer emits jamming signal $\mathbf{z} \in \mathbb{C}^{N_{j}}$ with distribution $\mathscr{C} \mathscr{N}(\mathbf{0}, \mathbf{Q})$ isotropically, where $\mathbf{Q}$ is the jamming covariance. Thus, the received signals at Bob and $k$-th Eve are, respectively, given by

$$
\begin{gathered}
y_{b}=\sqrt{P_{s}} \mathbf{g}_{b}^{H} \mathbf{D}(\mathbf{h}) \mathbf{w} s+\mathbf{n}_{r}^{T} \mathbf{D}^{H}\left(\mathbf{g}_{b}\right) \mathbf{w}+\mathbf{g}_{b, j}^{H} \mathbf{z}+n_{b}, \\
y_{e, k}=\sqrt{P_{s}} \mathbf{g}_{k}^{H} \mathbf{D}(\mathbf{h}) \mathbf{w} s+\mathbf{n}_{r}^{T} \mathbf{D}^{H}\left(\mathbf{g}_{k}\right) \mathbf{w}+\mathbf{g}_{k, j}^{H} \mathbf{z}+n_{k},
\end{gathered}
$$

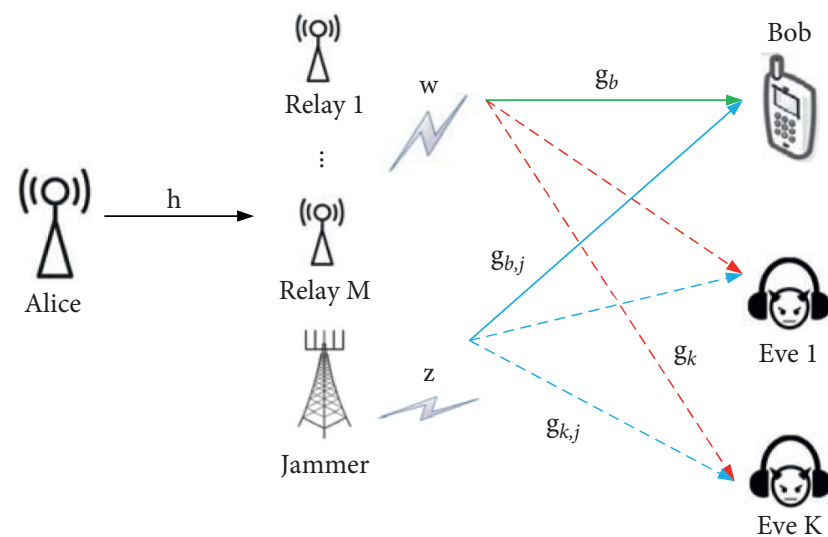

FIgURE 1: The secrecy relay system model with $\mathrm{CB}$ and jamming.

where $n_{b}$ and $n_{k}$ are additive noises at Bob and $k$-th Eve, with variance $\sigma_{b}^{2}$ and $\sigma_{k}^{2}$, respectively.

The signal-to-interference-plus-noise ratios (SINRs) at Bob and $k$-th Eve can be written as

$$
\begin{gathered}
\Gamma_{b} \triangleq \frac{\mathbf{w}^{H} \mathbf{A}_{b} \mathbf{w}}{\mathbf{w}^{H} \mathbf{B}_{b} \mathbf{w}+\mathbf{g}_{b, j}^{H} \mathbf{Q g _ { b , j }}+\sigma_{b}^{2}}, \\
\Gamma_{e, k} \triangleq \frac{\mathbf{w}^{H} \mathbf{A}_{k} \mathbf{w}}{\mathbf{w}^{H} \mathbf{B}_{k} \mathbf{w}+\mathbf{g}_{k, j}^{H} \mathbf{Q g} g_{k, j}+\sigma_{k}^{2}}, \quad \forall k \in \mathscr{K},
\end{gathered}
$$

where $\quad \mathbf{A}_{b}=P_{s} \mathbf{D}^{H}(\mathbf{h}) \mathbf{g}_{b} \mathbf{g}_{b}^{H} \mathbf{D}(\mathbf{h}), \quad \mathbf{B}_{b}=\sigma_{r}^{2} \mathbf{D}\left(\mathbf{g}_{b}\right) \mathbf{D}^{H}\left(\mathbf{g}_{b}\right)$, $\mathbf{A}_{k}=P_{s} \mathbf{D}^{H}(\mathbf{h}) \mathbf{g}_{k} \mathbf{g}_{k}^{H} \mathbf{D}(\mathbf{h}), \quad$ and $\quad \mathbf{B}_{k}=\sigma_{r}^{2} \mathbf{D}\left(\mathbf{g}_{k}\right) \mathbf{D}^{H}\left(\mathbf{g}_{k}\right)$, $\forall k \in \mathscr{K}$, respectively.

Accordingly, the achievable secrecy rate for the relay network is given by

$$
R=\min _{\forall k \in \mathscr{K}} f_{k}(\mathbf{w}, \mathbf{Q}),
$$

where $f_{k}(\mathbf{w}, \mathbf{Q})$ is the difference between the information rates for Bob and $k$-th Eve, which can be expressed as

$$
f_{k}(\mathbf{w}, \mathbf{Q})=C_{b}(\mathbf{w}, \mathbf{Q})-C_{e, k}(\mathbf{w}, \mathbf{Q}),
$$

where $C_{b}$ and $C_{e}$ are given as

$$
\begin{aligned}
C_{b}(\mathbf{w}, \mathbf{Q}) & =\frac{1}{2} \log \left(1+\Gamma_{b}\right), \\
C_{e, k}(\mathbf{w}, \mathbf{Q}) & =\frac{1}{2} \log \left(1+\Gamma_{e, k}\right), \quad \forall k \in \mathscr{K} .
\end{aligned}
$$

2.2. Problem Formulation. Notably, we assume a practical scenario that the relays and jammer have perfect CSI of Bob but only imperfect CSI about Eves. Similar to [11], the channel error vectors are modeled as circularly symmetric complex Gaussian (CSCG) distribution, i.e.,

$$
\begin{aligned}
\mathscr{G}_{k} & =\left\{\mathbf{g}_{k} \mid \mathbf{g}_{k}=\bar{g}_{k}+\Delta \mathbf{g}_{k}, \Delta \mathbf{g}_{k} \sim \mathscr{C} \mathcal{N}\left(\mathbf{0}, \mathbf{C}_{k}\right)\right\}, \\
\mathscr{G}_{k, j} & =\left\{\mathbf{g}_{k, j} \mid \mathbf{g}_{k, j}=\bar{g}_{k, j}+\Delta \mathbf{g}_{k, j}, \Delta \mathbf{g}_{k, j} \sim \mathscr{C} \mathcal{N}\left(\mathbf{0}, \mathbf{C}_{k, j}\right)\right\},
\end{aligned}
$$


where $\bar{g}_{k}$ and $\bar{g}_{k, j}$ are the estimated channel vectors, respectively, and $\Delta \mathbf{g}_{k}$ and $\Delta \mathbf{g}_{k, j}$ denote the channel uncertainty with covariance $\mathbf{C}_{k} \in \mathbb{U}_{+}^{M}$ and $\mathbf{C}_{k, j} \in \mathbb{U}_{+}^{N_{j}}$, respectively. In addition, $\mathbf{g}_{k}$ is independent of $\mathbf{g}_{l}$, for any $k \neq l$. Similarly, $\mathbf{g}_{k, j}$ is independent of $\mathbf{g}_{l, j}$, for any $k \neq l$.

In this paper, we assume that $\mathbf{C}_{k}=\varepsilon_{k}^{2} \mathbf{I}$ and $\mathbf{C}_{k, j}=\zeta_{k}^{2} \mathbf{I}$, with $\varepsilon_{k}^{2}$ and $\zeta_{k}^{2}$ denoting the channel uncertainty level. Besides, according to $[16,17]$, we consider two types of channel estimation error models:

(1) $\varepsilon_{k}^{2}$ and $\zeta_{k}^{2}$ are fixed and independent of the average SNR.

(2) $\varepsilon_{k}^{2}$ and $\zeta_{k}^{2}$ are functions of the average SNR. Take $\varepsilon_{k}^{2}$ as example. The variance of the error can be modeled as $\varepsilon_{k}^{2}=\Omega_{k} /\left(1+\Phi \gamma \Omega_{k}\right)$, where $\Omega_{k}=d^{-\alpha}$ is the variance of $\mathbf{g}_{k}$, e.g., $\mathbb{E}\left[\mathbf{g}_{k} \mathbf{g}_{k}^{H}\right]=\Omega_{k} \mathbf{I}$, where $d$ denotes the link distance, and $\alpha$ is the attenuation factor. In addition, $\gamma$ is the transmit SNR, and $\omega>0$ depends on the cost of acquiring CSI in light of the training pilot power consumption and reflects the quality of channel estimation.

In this paper, we aim to maximize the outage secrecy rate via jointly designing the $C B$ vector $\mathbf{w}$ and jamming covariance Q. Specifically, the OCSRM problem is formulated as

$$
\begin{array}{ll}
\max _{\mathbf{w}, \mathbf{Q}, R_{s}} & R_{s}, \\
\text { s.t. } & \underset{\mathbf{g}_{k}, \mathbf{g}_{k, j}}{\operatorname{Pr}}\left\{\min _{\forall k \in \mathscr{K}} f_{k}(\mathbf{w}, \mathbf{Q}) \geq R_{s}\right\} \geq 1-\delta, \\
& \mathbf{w}^{H} \mathbf{F} \mathbf{w}+\operatorname{Tr}(\mathbf{Q}) \leq P_{T}, \\
& \mathbf{w}^{H} \mathbf{F} \mathbf{w} \leq P_{R}, \\
& \operatorname{Tr}(\mathbf{Q}) \leq P_{J},
\end{array}
$$

where $\delta$ is the secrecy outage probability, e.g., a parameter denoting the chance of $R$ falling below the target rate $R_{s}$ due to CSI uncertainty. Apparently, $R_{s}$ is the outage secrecy rate. In addition, $\mathbf{F}=P_{s} \mathbf{D}^{H}(\mathbf{h}) \mathbf{D}(\mathbf{h})+\sigma_{r}^{2} \mathbf{I}$, and $P_{T}$ denotes the sum power budget for the relays and jammer, while $P_{R}$ and $P_{J}$ stand for the individual power budget for the relays and the jammer, respectively.

It should be noted that (7a)-(7e) are nonconvex due to the coupled variables and constraints. Hence, in the following, we will propose an effective way to convert $(7 a)-(7 e)$ into a solvable problem.

\section{An SDR-Based Approach to the OCSRM Problem}

In this section, we will derive an SDR-based approach to (7a)-(7e). Firstly, we decouple the probabilistic constraint in (7b). Since $\mathbf{g}_{k}, \mathbf{g}_{l}, l \neq k$ and $\mathbf{g}_{k, j}, \mathbf{g}_{l, j}, l \neq k$ are independent, we obtain

$$
(7 b) \Leftarrow \operatorname{Pr}_{\mathbf{g}_{k, \mathbf{g}_{k, j}}}\left\{f_{k}(\mathbf{w}, \mathbf{Q}) \geq R_{s}\right\} \geq 1-\bar{\delta}, \quad \forall k,
$$

where $\bar{\delta}=1-(1-\delta)^{1 / K}$ (see more details in [8]).

3.1. Relaxation of the OCSRM Problem. In this part, we will propose a two-layer optimization way to (7a)-(7e). To start with, we rewrite (7a)-(7e) as

$$
\begin{aligned}
& R_{s}^{*}=\max _{\mathbf{w}, \mathbf{Q} \geqslant \mathbf{0}, \beta \geq 1} C_{b}(\mathbf{w}, \mathbf{Q})-\log \beta, \\
& \text { s.t. } \underset{\mathbf{g}_{k}, \mathbf{g}_{k, j}}{\operatorname{Pr}}\left\{C_{e, k}(\mathbf{w}, \mathbf{Q}) \leq \log \beta\right\} \geq 1-\bar{\delta}, \quad \forall k \in \mathscr{K}, \\
& (7 b)-(7 d),
\end{aligned}
$$

where $\beta$ is an introduced slack variable to simplify the objective function.

By substituting (2a) and (2b) into (9a)-(9c), we reexpress $(9 a)-(9 c)$ as

$$
R_{s}^{*}=\max _{\mathbf{w}, \mathbf{Q} \geqslant \mathbf{0}, \beta \geq 1} \log \left(1+\frac{\mathbf{w}^{H} \mathbf{A}_{b} \mathbf{w}}{\mathbf{w}^{H} \mathbf{B}_{b} \mathbf{w}+\mathbf{g}_{b, j}^{H} \mathbf{Q g} \mathbf{g}_{b, j}+\sigma_{b}^{2}}\right)-\log \beta,
$$

$$
\begin{aligned}
& \text { s.t. } \underset{\mathbf{g}_{k, \mathbf{g}_{k, j}}}{\operatorname{Pr}}\left\{1+\frac{\mathbf{w}^{H} \mathbf{A}_{k} \mathbf{w}}{\mathbf{w}^{H} \mathbf{B}_{b} \mathbf{w}+\mathbf{g}_{k, j}^{H} \mathbf{Q g}_{k, j}+\sigma_{k}^{2}} \leq \beta\right\} \\
& \geq 1-\bar{\delta}, \quad \forall k \in \mathscr{K}, \\
& (7 b)-(7 d) .
\end{aligned}
$$

Furthermore, utilizing the SDR [18], e.g., defining $\mathbf{W}=$ $\mathbf{w w}^{H}$ and neglecting the nonconvex constraint $\operatorname{rank}(\mathbf{W})=1$, we attain the following SDR problem:

$$
\begin{array}{ll}
\text { s.t. } & \operatorname{Pr}_{\mathbf{g}_{k}, \mathbf{g}_{k, j}}\left\{(\beta-1)\left(\operatorname{Tr}\left(\mathbf{B}_{k} \mathbf{W}\right)+\mathbf{g}_{k, j}^{H} \mathbf{Q g}_{k, j}+\sigma_{k}^{2}\right)\right. \\
\left.\geq \operatorname{Tr}\left(\mathbf{A}_{k} \mathbf{W}\right)\right\} \geq 1-\bar{\delta}, \quad \forall k \in \mathscr{K}, \\
\operatorname{Tr}(\mathbf{F W})+\operatorname{Tr}(\mathbf{Q}) \leq P_{T}, \\
\operatorname{Tr}(\mathbf{F W}) \leq P_{R}, \\
\operatorname{Tr}(\mathbf{Q}) \leq P_{J} .
\end{array}
$$$$
R_{s}^{*}=\max _{\substack{\mathbf{W} \geqslant \mathbf{Q}, \mathbf{Q} 0 \mathbf{0}, \beta \geq 1}} \log \left(1+\frac{\operatorname{Tr}\left(\mathbf{A}_{b} \mathbf{W}\right)}{\sigma_{b}^{2}+\operatorname{Tr}\left(\mathbf{B}_{b} \mathbf{W}\right)+\mathbf{g}_{b, j}^{H} \mathbf{Q} \mathbf{g}_{b, j}}\right)-\log \beta,
$$

3.2. An SDR-Based Line Search Method for (11a)-(11e). We now focus on solving the relaxation (11a)-(11e). In fact, (11a)-(11e) can be reformulated as a single-variable optimization problem, which can be efficiently handled by SDR. To show this, note that 


$$
\begin{aligned}
1 & \leq \beta \leq 1+\frac{\mathbf{w}^{H} \mathbf{A}_{b} \mathbf{w}}{\sigma_{b}^{2}+\mathbf{w}^{H} \mathbf{B}_{b} \mathbf{w}+\mathbf{g}_{b, j}^{H} \mathbf{Q} \mathbf{g}_{b, j}} \\
& \leq 1+\frac{\mathbf{w}^{H} \mathbf{A}_{b} \mathbf{w}}{\mathbf{w}^{H} \mathbf{B}_{b} \mathbf{w}} \leq 1+\lambda_{\max }\left(\mathbf{A}_{b}, \mathbf{B}_{b}\right) .
\end{aligned}
$$

Then, we rewrite (11a)-(11e) as

$$
\begin{aligned}
& e^{R_{s}^{*}}=\max _{\alpha} \varphi(\alpha), \\
& \text { s.t. }\left(1+\lambda_{\max }\left(\mathbf{A}_{b}, \mathbf{B}_{b}\right)\right)^{-1} \leq \alpha \leq 1,
\end{aligned}
$$

where $\alpha=1 / \beta$ and

$$
\begin{aligned}
& \varphi(\alpha)=\max _{\mathbf{W} \geqslant \mathbf{0}, \mathbf{Q} \geqslant 0} \frac{\sigma_{b}^{2}+\operatorname{Tr}\left(\mathbf{B}_{b} \mathbf{W}\right)+\mathbf{g}_{b, j}^{H} \mathbf{Q g}_{b, j}+\operatorname{Tr}\left(\mathbf{A}_{b} \mathbf{W}\right)}{\alpha^{-1}\left(\operatorname{Tr}\left(\mathbf{B}_{b} \mathbf{W}\right)+\mathbf{g}_{b, j}^{H} \mathbf{Q g}_{b, j}+\sigma_{b}^{2}\right)}, \\
& \text { s.t. } \operatorname{Pr}_{\mathbf{g}_{k, \mathbf{g}_{k, j}}}\left\{\left(\alpha^{-1}-1\right)\left(\operatorname{Tr}\left(\mathbf{B}_{k} \mathbf{W}\right)+\mathbf{g}_{k, j}^{H} \mathbf{Q g}_{k, j}+\sigma_{k}^{2}\right)\right. \\
& \left.\quad \geq \operatorname{Tr}\left(\mathbf{A}_{k} \mathbf{W}\right)\right\} \geq 1-\bar{\delta}, \quad \forall k \in \mathscr{K}, \\
& (11 c)-(11 e) .
\end{aligned}
$$

$\varphi(\alpha)$ does not have a closed form but is numerically tractable. In particular, (14a)-(14c) can be converted into a convex optimization problem. Specifically, via the Charnes-Cooper transformation [19], we introduce a change of variables $\mathbf{W}=\widetilde{\mathbf{W}} / \xi, \mathbf{Q}=\widetilde{\mathbf{Q}} / \xi$ with $\xi>0$. Then, (14a)-(14c) can be equivalently expressed as

$$
\varphi(\alpha)=\max _{\widetilde{\mathbf{W}} \succcurlyeq \mathbf{0}, \widetilde{\mathbf{Q}} \geqslant \mathbf{0}, \xi>0} \xi \sigma_{b}^{2}+\operatorname{Tr}\left(\mathbf{B}_{b} \tilde{W}\right)+\mathbf{g}_{b, j}^{H} \widetilde{Q} \mathbf{g}_{b, j}+\operatorname{Tr}\left(\mathbf{A}_{b} \tilde{W}\right),
$$

$$
\text { s.t. } \operatorname{Tr}\left(\mathbf{B}_{b} \mathbf{W}\right)+\mathbf{g}_{b, j}^{H} \widetilde{Q} \mathbf{g}_{b, j}+\xi \sigma_{b}^{2}=\alpha \text {, }
$$

$$
\begin{aligned}
& \operatorname{Pr}_{\mathbf{g}_{k, \mathbf{g}_{k, j}}}\left\{(1-\alpha)\left(\operatorname{Tr}\left(\mathbf{B}_{k} \widetilde{W}\right)+\mathbf{g}_{k, j}^{H} \widetilde{Q} \mathbf{g}_{k, j}+\xi \sigma_{k}^{2}\right)\right. \\
& \left.\quad \geq \alpha \operatorname{Tr}\left(\mathbf{A}_{k} \widetilde{W}\right)\right\} \geq 1-\bar{\delta}, \quad \forall k \in \mathscr{K} \\
& \operatorname{Tr}(\mathbf{F} \tilde{W})+\operatorname{Tr}(\widetilde{Q}) \leq \xi P_{T}, \\
& \operatorname{Tr}(\mathbf{F} \tilde{W}) \leq \xi P_{R},
\end{aligned}
$$$$
\operatorname{Tr}(\widetilde{Q}) \leq \xi P_{J}
$$

3.3. Transformation of Probabilistic Constraints (15c). The remaining challenging is that $(15 \mathrm{a})-(15 \mathrm{f})$ have probabilistic constraints (15c), which is intractable. In the following, we will handle $(15 \mathrm{c})$ via the BTI and LDI, respectively.

Firstly, using the following identity $\mathbf{a}^{H} \mathbf{D}(\mathbf{b})=\mathbf{b}^{H} \mathbf{D}(\mathbf{a})$, we obtain $\operatorname{Tr}\left(\mathbf{A}_{k} \widetilde{W}\right)=\mathbf{g}_{k}^{H} \mathbf{H} \tilde{W} \mathbf{H}^{H} \mathbf{g}_{k}$ and $\operatorname{Tr}\left(\mathbf{B}_{k} \widetilde{W}\right)=\mathbf{g}_{k}^{H}\left(\sigma_{r}^{2} \mathbf{I}\right.$ $\odot \tilde{W}) \mathbf{g}_{k}$.

Then, we transform (15a)-(15f) into

$$
\varphi(\alpha)=\max _{\widetilde{W} \succcurlyeq \mathbf{0}, \widetilde{Q} \succcurlyeq \mathbf{0}, \xi>0} \xi \sigma_{b}^{2}+\operatorname{Tr}\left(\mathbf{B}_{b} \widetilde{W}\right)+\mathbf{g}_{b, j}^{H} \widetilde{Q} \mathbf{g}_{b, j}+\operatorname{Tr}\left(\mathbf{A}_{b} \tilde{W}\right),
$$

$$
\begin{array}{ll}
\text { s.t. } & \operatorname{gr}_{\mathbf{g}_{k,} \mathbf{g}_{k, j}}\left\{\mathbf{g}_{k}^{H} \mathbf{X} \mathbf{g}_{k}+(1-\alpha) \mathbf{g}_{k, j}^{H} \widetilde{Q} \mathbf{g}_{k, j}+(1-\alpha) \xi \sigma_{k}^{2} \geq 0\right\} \\
& \geq 1-\bar{\delta}, \quad \forall k \in \mathscr{K},
\end{array}
$$

$(15 b),(15 d)-(15 f)$,

where $\mathbf{X}=(1-\alpha)\left(\sigma_{r}^{2} \mathbf{I}\right) \odot \widetilde{W}-\alpha P_{s} \mathbf{H} \tilde{W} \mathbf{H}^{H}$.

It should be noted that the outage probability in (16b) can be characterized by the quadratic inequality with respect to (w.r.t.) $\mathbf{g}_{k}$ and $\mathbf{g}_{k, j}$, e.g., (16b) can be safely approximated via the following BTI and LDI, respectively.

Lemma 1 (BTI [11]). For any $(\mathbf{A}, \mathbf{u}, c) \in \mathbb{H}^{N} \times \mathbb{C}^{N} \times \mathbb{R}$, $\mathbf{v} \sim \mathscr{C} \mathcal{N}(\mathbf{0}, \mathbf{I})$, and $\eta \in(0,1]$, the following inequalities hold:

$$
\begin{aligned}
& \operatorname{Pr}_{\mathbf{v}}\left\{\mathbf{v}^{H} \mathbf{A v}+2 \mathfrak{R}\left\{\mathbf{v}^{H} \mathbf{u}\right\}+c \geq 0\right\} \geq 1-\eta, \\
& \Leftarrow\left\{\begin{array}{l}
\operatorname{Tr}(\mathbf{A})-\sqrt{-2 \ln (\eta)} x+\ln (\eta) y+c \geq 0, \\
\left\|\left[\begin{array}{c}
\operatorname{vec}(\mathbf{A}) \\
\sqrt{2} \mathbf{u}
\end{array}\right]\right\| \leq x, \\
y \mathbf{I}+\mathbf{A} \geqslant \mathbf{0}, y \geq 0,
\end{array}\right.
\end{aligned}
$$

where $x$ and $y$ are slack variables. Moreover, (17) is convex w.r.t. all the variables $(\mathbf{A}, \mathbf{u}, c, x, y)$.

To use Lemma 1, we first define $\mathbf{g}_{k}=\bar{g}_{k}+\mathbf{C}_{k}^{1 / 2} \mathbf{e}_{k}$ and $\mathbf{g}_{k, j}=\bar{g}_{k, j}+\mathbf{C}_{k, j}^{1 / 2} \mathbf{e}_{k, j}$, where $\quad \mathbf{e}_{k} \sim \mathscr{C} \mathscr{N}(\mathbf{0}, \mathbf{I}) \quad$ and $\mathbf{e}_{k, j} \sim \mathscr{C} \mathcal{N}(\mathbf{0}, \mathbf{I})$, respectively.

Then, the part in the braces of (16b) can be equivalently rewritten as

$$
\begin{aligned}
\mathbf{e}_{k}^{H} & \mathbf{C}_{k}^{1 / 2} \mathbf{X} \mathbf{C}_{k}^{1 / 2} \mathbf{e}_{k}+2 \Re\left\{\mathbf{e}_{k}^{H} \mathbf{C}_{k}^{1 / 2} \mathbf{X} \bar{g}_{k}\right\}+\bar{g}_{k}^{H} \mathbf{X} \bar{g}_{k} \\
& +(1-\alpha) \xi \sigma_{k}^{2}+(1-\alpha) \mathbf{e}_{k, j}^{H} \mathbf{C}_{k, j}^{1 / 2} \widetilde{\mathbf{Q C}}_{k, j}^{1 / 2} \mathbf{e}_{k, j} \\
& +2(1-\alpha) \Re\left\{\mathbf{e}_{k, j}^{H} \mathbf{C}_{k}^{1 / 2} \widetilde{Q}_{k, j}\right\}+(1-\alpha) \bar{g}_{k, j}^{H} \widetilde{Q}_{k} \bar{g}_{k, j} \geq 0 .
\end{aligned}
$$

Furthermore, (18) can be rewritten as

$$
\mathbf{v}_{k}^{H} \mathbf{A}_{k} \mathbf{v}_{k}+2 \mathfrak{R}\left\{\mathbf{v}_{k}^{H} \mathbf{u}_{k}\right\}+c_{k} \geq 0,
$$

where $\mathbf{A}_{k}, \mathbf{v}_{k}, \mathbf{u}_{k}$, and $c_{k}$ are given as

$$
\begin{aligned}
& \mathbf{A}_{k}=\left[\begin{array}{c}
\mathbf{C}_{k}^{1 / 2} \mathbf{X C}_{k}^{1 / 2} \mathbf{0} \\
\mathbf{0}(1-\alpha) \mathbf{C}_{k, j}^{1 / 2} \widetilde{\mathbf{Q C}}_{k, j}^{1 / 2}
\end{array}\right], \\
& \mathbf{v}_{k}=\left[\mathbf{e}_{k}^{H}, \mathbf{e}_{k, j}^{H}\right], \\
& \mathbf{u}_{k}=\left[\begin{array}{c}
\mathbf{C}_{k}^{1 / 2} \mathbf{X} 0 \\
\mathbf{0}(1-\alpha) \mathbf{C}_{k}^{1 / 2} \widetilde{Q}
\end{array}\right]\left[\begin{array}{c}
\bar{g}_{k} \\
\bar{g}_{k, j}
\end{array}\right], \\
& c_{k}=\left[\overline{\mathfrak{g}}_{k}^{H}, \overline{\mathfrak{g}}_{k, j}^{H}\right]\left[\begin{array}{c}
\mathbf{X} 0 \\
\mathbf{0}(1-\alpha) \widetilde{Q}
\end{array}\right]\left[\begin{array}{c}
\bar{g}_{k} \\
\bar{g}_{k, j}
\end{array}\right]+(1-\alpha) \xi \sigma_{k}^{2} .
\end{aligned}
$$


Combining all these equations, (16a)-(16c) can be recast as the following convex problem:

$$
\begin{aligned}
& \begin{array}{c}
\varphi(\alpha)=\max _{\tilde{\mathbf{w}} \geqslant \mathbf{0}, \widetilde{\mathbf{Q}} \geqslant \mathbf{0}, \xi>0, y_{k}>0} \xi \sigma_{b}^{2}+\operatorname{Tr}\left(\mathbf{B}_{b} \widetilde{W}\right) \\
+\mathbf{g}_{b, j}^{H} \widetilde{\mathbf{Q}} \mathbf{g}_{b, j}+\operatorname{Tr}\left(\mathbf{A}_{b} \tilde{W}\right),
\end{array} \\
& \text { s.t. } \operatorname{Tr}\left(\mathbf{A}_{k}\right)-\sqrt{-2 \ln (\bar{\delta})} x_{k} \\
& +\ln (\bar{\delta}) y_{k}+c_{k} \geq 0, \quad \forall k \in \mathscr{K}, \\
& \left\|\left[\begin{array}{c}
\operatorname{vec}\left(\mathbf{A}_{k}\right) \\
\sqrt{2} \mathbf{u}_{k}
\end{array}\right]\right\| \leq x_{k}, \quad \forall k \in \mathscr{K}, \\
& y_{k} \mathbf{I}+\mathbf{A}_{k} \geqslant \mathbf{0}, \quad y_{k} \geq 0, \forall k \in \mathscr{K}, \\
& (15 b),(15 d)-(15 f),(20),
\end{aligned}
$$

which can be efficiently solved by available solvers such as CVX [20].

It should be mentioned that for the obtained $\mathbf{W}^{*}$, if $\operatorname{rank}\left(\mathbf{W}^{*}\right)=1$, we can attain the optimal $\mathbf{w}^{*}$ via matrix eigenvector decomposition. On the other hand, if $\operatorname{rank}\left(\mathbf{W}^{*}\right) \geq 1$, we employ the Gaussian randomization (GR) method to generate the suboptimal rank-one solution [18].

In the above part, we have utilized the BTI to transform the probabilistic constraint. To reduce the computational complexity, in the following we will utilize the LDI to convert the probabilistic constraint.

Lemma 2 (LDI [11]). Let $\mathbf{x} \sim \mathscr{C} \mathcal{N}(\mathbf{0}, \mathbf{I})$, and let $\mathbf{A} \in \mathbb{Q}^{n \times n}$, $\mathbf{r} \in \mathbb{C}^{n \times 1}$ be given variables. Then, for any $v>1 / \sqrt{2}$ and $\zeta>0$, we have

$$
\begin{aligned}
\operatorname{Pr}\left\{\mathbf{x}^{H} \mathbf{A} \mathbf{x}+2 \mathfrak{R}\left\{\mathbf{x}^{H} \mathbf{r}\right\}\right. & \leq \operatorname{Tr}(\mathbf{A})-\zeta\} \\
& \leq \begin{cases}\exp \left(-\frac{\zeta^{2}}{4 T^{2}}\right), & 0<\zeta \leq 2 \bar{v} v T, \\
\exp \left(-\frac{\bar{v} v \zeta}{T}+(\bar{v} v)^{2}\right), & \zeta>2 \bar{v} v T,\end{cases}
\end{aligned}
$$

where $\bar{v}=1-\left(1 / 2 v^{2}\right)$ and $T=v\|\mathbf{A}\|+1 / \sqrt{2}\|\mathbf{r}\|$.

By Lemma 2 and following the similar way in [11], (16b) can be transformed into the following relationships:

$$
\left\{\begin{array}{l}
\operatorname{Tr}\left(\mathbf{A}_{k}\right)+c_{k} \geq 2 \sqrt{-\ln (\bar{\delta})}\left(x_{k}+y_{k}\right), \quad \forall k \in \mathscr{K}, \\
\left\|\mathbf{u}_{k}\right\| \leq \sqrt{2} x_{k}, \quad \forall k \in \mathscr{K}, \\
v\left\|\mathbf{A}_{k}\right\| \leq y_{k}, \quad \forall k \in \mathscr{K},
\end{array}\right.
$$

where $v>1 / \sqrt{2}$ is the solution to $\left(1-\left(1 /\left(2 v^{2}\right)\right)\right)$ $v=\sqrt{-\ln (\bar{\delta})}$ (see more details in reference [11]).

Thus, (16a)-(16c) can be recast as the following convex problem:

$$
\begin{aligned}
& \varphi(\alpha)=\max _{\widetilde{\mathbf{W}} \geqslant \mathbf{0}, \widetilde{\mathbf{Q}} \geqslant \mathbf{0}, \xi>0, x_{k}, y_{k}} \xi \sigma_{b}^{2}+\operatorname{Tr}\left(\mathbf{B}_{b} \widetilde{W}\right) \\
& +\mathbf{g}_{b, j}^{H} \widetilde{Q} \mathbf{g}_{b, j}+\operatorname{Tr}\left(\mathbf{A}_{b} \widetilde{W}\right), \\
& \text { s.t. }(15 b),(15 d)-(15 f),(20),(23) \text {, }
\end{aligned}
$$

which can also be solved by CVX. Similar to (21a), GR is utilized to extract the rank-one solution.

Following the theory in [11], we obtain the complexity comparison between the BTI and LDI methods, which are shown in Table 1, where $\vartheta$ denotes the accuracy requirement. From the comparison, we can see that the LDI method achieves lower complexity than the BTI method.

\section{Simulation Results}

In this section, simulation results are presented to evaluate the performance of the proposed design. The channels $\mathbf{h}, \mathbf{g}_{b}$, $\mathbf{g}_{k}, \mathbf{g}_{b, j}$, and $\mathbf{g}_{k, j}$ are assumed to be independent and identically distributed complex Gaussian random variables with zero mean and unit variance. We set $K=3, M=5, N_{j}=3$, $P_{s}=10 \mathrm{~dB}, P_{T}=10 \mathrm{~dB}, \sigma_{r}^{2}=\sigma_{b}^{2}=\sigma_{e, k}^{2}=1, \alpha=4, d=20$, $\emptyset=0.2$, and $\delta=10^{-2}$. In addition, we compare our design with the following methods: (1) the proposed design in the case of perfect Eves' CSI, which can be seen as the upper bound of the robust design; (2) the no jam case, e.g., setting $\mathbf{Q}=\mathbf{0}$ while only optimizing $\mathbf{w}$; and (3) the fixed CSI uncertainty model with BTI method, and $\varepsilon_{k}^{2}=\zeta_{k}^{2}=10^{-4}$. These methods are denoted as "BTI method," "LDI method," "perfect CSI case," "no jam method," and "fixed CSI uncertainty," respectively.

Firstly, we compare the outage secrecy rates versus the total power budget $P_{T}$, and the result is shown in Figure 2 . From this figure, we can see that the outage secrecy rates increase with the increase of $P_{T}$, while the BTI method outperforms the LDI method and the no jam method. In addition, the performance gap between perfect and imperfect CSI cases is apparent, especially for relative large $P_{T}$, which shows the influence of the CSI error on the security. Besides, the fixed CSI uncertainty model suffers certain performance loss, especially for relative large $P_{T}$, since when $P_{T}$ tends to large, according to the function model, the corresponding CSI tends to smaller. However, the fixed CSI uncertainty model cannot reflect this relationship, thus leading to performance loss.

Next, we investigate the effect of the number of the relays on the outage secrecy rate. Figure 3 shows the relationship between the outage secrecy rate and the number of the relays. From this figure, we can see that for all these methods, higher outage secrecy rate can be achieved with more relays, since higher spatial degrees of freedom can be utilized to improve the secrecy performance.

Lastly, Figure 4 investigates the relationship between the outage secrecy rate versus the secrecy outage probability. As can be seen in this figure, with the increase of $\delta$, the outage secrecy rate tends to increase. This is mainly due to the fact that with the increase of $\delta$, the relay network 
TABLE 1: Complexity comparison of the BTI and LDI methods.

\begin{tabular}{lc}
\hline Methods & Complexity order $(\ln (1 / \mathcal{\vartheta}) \sqrt{\beta(\mathscr{X})} \mathscr{C})$ \\
\hline BTI & $\beta(\mathscr{X})=5+3 K+K M, \mathscr{C}=n\left(5+3 K+K M^{3}\right)$ \\
& $+n^{2}\left(5+3 K+K M^{2}\right)+n K\left(M^{2}+M+N_{j}\right)^{2}$ \\
& $+n^{3}$, and $n=\mathcal{O}\left(M^{2}+N_{j}^{2}+2 K+1\right)$. \\
& \\
LDI & $\beta(\mathscr{X})=5 K+5, \mathscr{C}=\left(n^{2}+n\right)(5 K+5)$ \\
& $+n K\left(M^{4}+M^{2}+2 M N_{j}+N_{j}^{2}\right)+n^{3}$, \\
& and $n=\mathscr{O}\left(M^{2}+N_{j}^{2}+2 K+1\right)$. \\
\hline
\end{tabular}

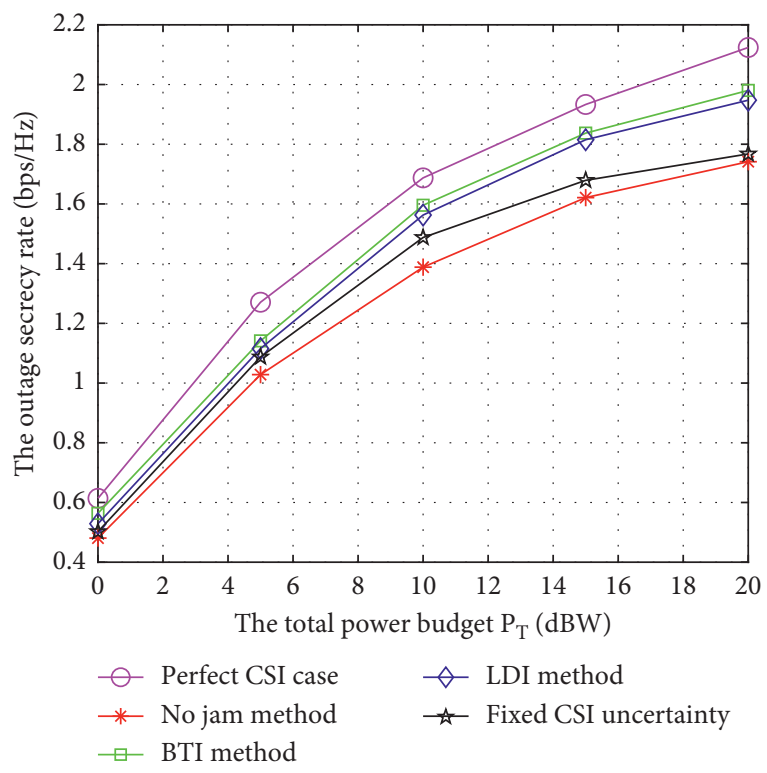

FIGURE 2: The outage secrecy rate versus the total power budget.

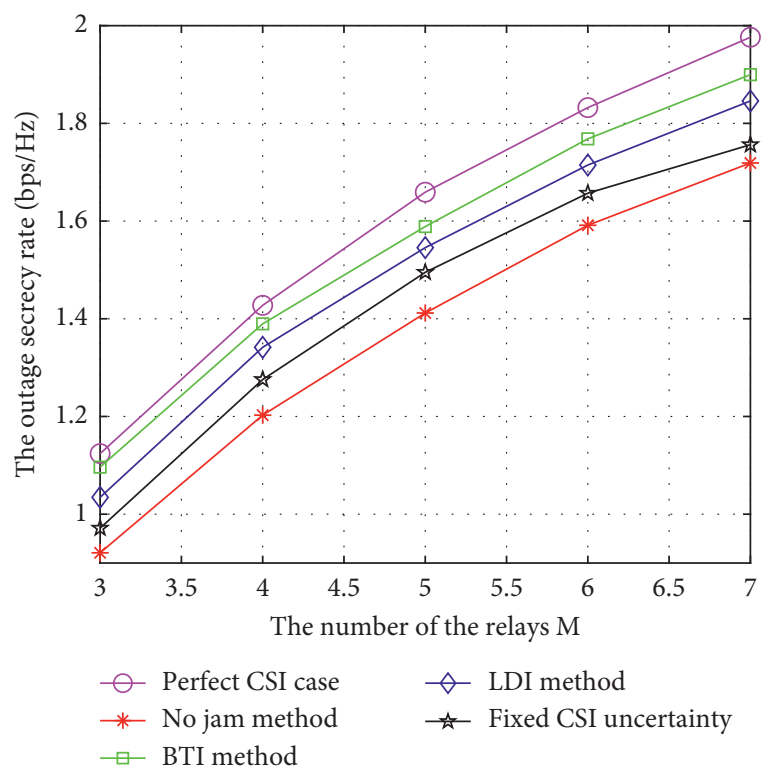

FIgURE 3: The outage secrecy rate versus the number of the relays.

permits more chance to outage, and thus the threshold of outage rate tends to increase, e.g., the outage secrecy rate tends to increase.

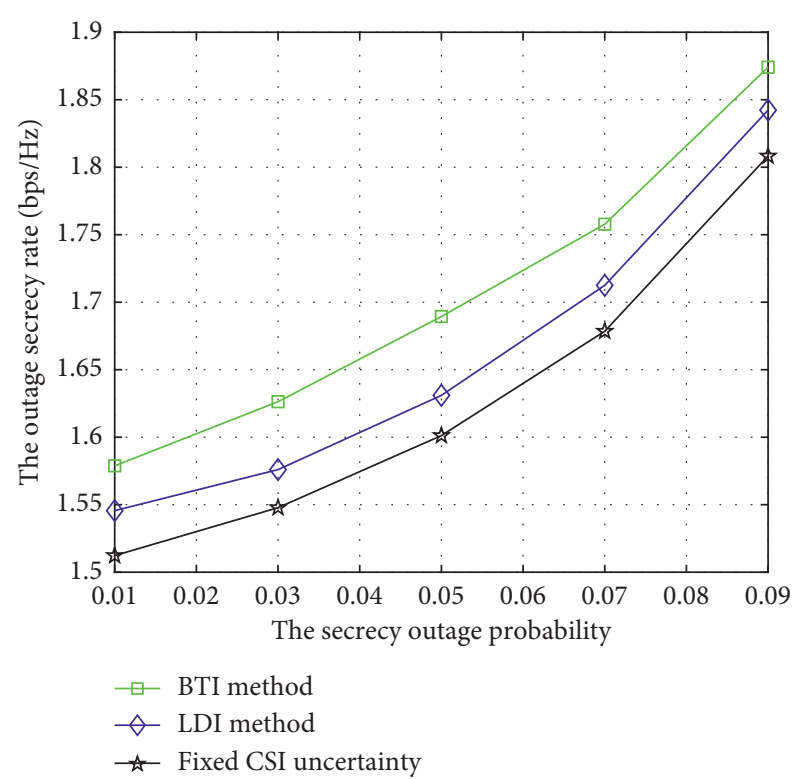

FIgURE 4: The outage secrecy rate versus the secrecy outage probability.

\section{Conclusion}

In this paper, we have investigated the OCSRM design in secrecy AF relay networks, via jointly optimizing the CB and jamming covariance. To solve the formulated nonconvex problem, we derived a safe approximation of the outage secrecy rate, and then a two-layer optimization algorithm was proposed. Simulation results demonstrated the performance of the proposed method.

\section{Data Availability}

The data used to support the findings of this study are available from the corresponding author upon request.

\section{Conflicts of Interest}

The authors declare that there are no conflicts of interest regarding the publication of this article.

\section{Acknowledgments}

This study was supported by the Industry-University-Research Cooperation Project of Jiangsu Province under grant no. BY2018282.

\section{References}

[1] Y. Liu, H.-H. Chen, and L. Wang, "Physical layer security for next generation wireless networks: theories, technologies, and challenges," IEEE Communications Surveys and Tutorials, vol. 19, no. 1, pp. 347-376, 2017.

[2] F. Jameel, S. Wyne, G. Kaddoum, and T. Q. Duong, “A comprehensive survey on cooperative relaying and jamming strategies for physical layer security," IEEE Communications Surveys and Tutorials, vol. 21, no. 3, pp. 2734-2771, 2019.

[3] E. R. Alotaibi and K. A. Hamdi, "Optimal cooperative relaying and jamming for secure communication," IEEE Wireless Communications Letters, vol. 4, no. 6, pp. 689-692, 2015. 
[4] H.-M. Wang, F. Liu, and M. Yang, "Joint cooperative beamforming, jamming, and power allocation to secure AF relay systems," IEEE Transactions on Vehicular Technology, vol. 64, no. 10, pp. 4893-4898, 2015.

[5] C. Wang, H.-M. Wang, and X.-G. Xia, "Hybrid opportunistic relaying and jamming with power allocation for secure cooperative networks," IEEE Transactions on Wireless Communications, vol. 14, no. 2, pp. 589-605, 2015.

[6] A. Behnad and X. Wang, "Jamming in dual-hop amplify-andforward relaying," IEEE Transactions on Vehicular Technology, vol. 64, no. 11, pp. 5020-5030, 2015.

[7] J. Xiong, L. Cheng, D. Ma, and J. Wei, "Destination-aided cooperative jamming for dual-hop amplify-and-forward MIMO untrusted relay systems," IEEE Transactions on Vehicular Technology, vol. 65, no. 9, pp. 7274-7284, 2016.

[8] Q. Li, W.-K. Ma, and A. M.-C. So, "A safe approximation approach to secrecy outage design for MIMO wiretap channels," IEEE Signal Processing Letters, vol. 21, no. 1, pp. 118-121, 2014.

[9] Z. Chu, H. Xing, M. Johnston, and S. Le Goff, "Secrecy rate optimizations for a MISO secrecy channel with multiple multiantenna eavesdroppers," IEEE Transactions on Wireless Communications, vol. 15, no. 1, pp. 283-297, 2016.

[10] J. Xu, S. Xu, and C. Xu, "Probabilistic robust secure beamforming in MISO channels with imperfect LCSI and statistical ECSI," IEEE Access, vol. 5, pp. 10277-10284, 2017.

[11] M. R. A. Khandaker, K.-K. Wong, Y. Zhang, and Z. Zheng, "Probabilistically robust SWIPT for secrecy MISOME systems," IEEE Transactions on Information Forensics and Security, vol. 12, no. 1, pp. 211-226, 2017.

[12] H. Niu, B. Zhang, and D. Guo, "Outage constrained robust beamforming and power splitting for secure MISO SWIPT channel," IEEE Wireless Communications Letters, vol. 6, no. 6, pp. 826-829, 2017.

[13] H. Niu, D. Guo, Y. Huang, B. Zhang, and B. Gao, "Outage constrained robust energy harvesting maximization for secure MIMO SWIPT systems," IEEE Wireless Communications Letters, vol. 6, no. 5, pp. 614-617, 2017.

[14] Y. Yuan and Z. Ding, "Outage constrained secrecy rate maximization design with SWIPT in MIMO-CR systems," IEEE Transactions on Vehicular Technology, vol. 67, no. 6, pp. 5475-5480, 2018.

[15] H. Song, H. Wen, R.-F. Liao, Y. Chen, and S. Chen, "Outage constrained secrecy rate maximization for MIMOME multicast wiretap channels," IEEE Wireless Communications Letters, vol. 8, no. 3, pp. 657-660, 2019.

[16] X. Li, J. Li, Y. Liu, Z. Ding, and A. Nallanathan, "Residual transceiver hardware impairments on cooperative NOMA networks," IEEE Transactions on Wireless Communications, vol. 19, no. 1, pp. 680-695, 2020.

[17] L. Wang, Y. Cai, and W. Yang, "On the finite-SNR DMT of two-way AF relaying with imperfect CSI," IEEE Wireless Communications Letters, vol. 1, no. 3, pp. 161-164, 2012.

[18] Z. Luo, W. Ma, and A. M.-C. So, "Semidefinite relaxation of optimization quadratic problems," IEEE Signal Processing Magazine, vol. 27, pp. 31-34, 2010.

[19] A. Charnes and W. W. Cooper, "Programming with linear fractional functionals," Naval Research Logistics Quarterly, vol. 9, no. 3-4, pp. 181-186, 1962.

[20] M. Grant and S. Boyd, "CVX: matlab software for disciplined convex programming, version 2.0 beta," 2013, http://cvxr. $\mathrm{com} / \mathrm{cvx}$. 